\title{
Single Pulse Calibration of Magnetic Field Sensors Using Mobile 43 kJ Facility
}

\author{
Audrius Grainys ${ }^{1}$, Jurij Novickij ${ }^{1}$, Tomaš Stankevič ${ }^{2}$, Voitech Stankevič ${ }^{1,2}$, Vitalij Novickij ${ }^{1}$, \\ Nerija Žurauskiené $\dot{1}^{1,2}$ \\ ${ }^{1}$ Vilnius Gediminas Technical University, Naugarduko 41, 03227, Vilnius, Lithuania; vitalij.novickij@vgtu.lt \\ ${ }^{2}$ Centre for Physical Sciences and Technology, Goštauto 11,01108, Vilnius, Lithuania; wstan@pfi.lt
}

\begin{abstract}
In this work we present a mobile $43 \mathrm{~kJ}$ pulsed magnetic field facility for single pulse calibration of magnetic field sensors. The magnetic field generator is capable of generating magnetic fields up to $40 \mathrm{~T}$ with pulse durations in the range of 0.3-2 ms. The high power crowbar circuit is used for the reverse voltage protection and pulse shaping purposes. The structure, the development challenges and the implemented solutions to improve the facility for the calibration of the magnetic field sensors are overviewed. The experimental data of the application of the proposed generator for the calibration of manganite magnetic field sensors are presented.
\end{abstract}

Keywords: Magnetic field measurement, calibration, sensor, high power.

\section{INTRODUCTION}

$\mathrm{I}_{\mathrm{m}}^{\mathrm{N}}$ THE LAST few decades interest in the high-pulsed magnetic field generation and the magnetic field measurements has increased dramatically due to the wide application range of the pulsed power technologies in science, medicine, military, and the industry. At the same time the sensors that are used for the measurement of the alternating high magnetic field have to satisfy the following requirements: the absence of saturation at high magnetic fields and high-speed responsiveness [1, 2].

In many sensors a drift is developed over a long period of time due to aging, contamination or temperature influence [3]. Combined with the possible non-linearity of the response a need for the systematic calibration procedures is formed. Typical calibration setups include an electromagnet for the magnetic field generation; however, such setups are usually limited in the 2-3 T range due to the core saturation. Further increase of the current in the electromagnet introduces Joule heating management issues, the setup requires the high efficacy active cooling - the size, complexity and the price of the facility are increased dramatically [4].

A common solution to generate the high pulsed magnetic fields is to discharge the high power capacitors through the inductor, which is connected as a load of the generator [510]. The semiconductor switches are used for the pulse forming purposes [7-10]. As a result a large variety of unique high power setups have been developed for the experiments in the magnetic fields [5-10]. However, such systems are usually high cost and stationary due to their size and weight $[5,7]$. The size of the magnetic field systems is heavily influenced by the amount of the accumulated and discharged energy [5, 7]. It is imminent that a trade-off between the power, size and the effective volume of the effect must be achieved during the development of a magnetic field system. The discharged energy and the effective volume limitations also introduce restraints for the applicability of the setup, however, at the same time the mobility of the pulsed magnetic field system can be achieved. The limitation of the total power to the specific needs of the experiments reduces the total price of the setup.

In this work we propose a mobile low-cost facility for pulsed magnetic field generation up to $40 \mathrm{~T}$ for a single pulse calibration of magnetic field sensors.

\section{SUBJECT \& METHODS}

\section{A. Estimation of system parameters}

The main requirements for the pulsed magnetic field facility were the mobility, safety, the magnetic field pulse amplitude up to $40 \mathrm{~T}$ and the pulse duration from $300 \mu$ s to $2 \mathrm{~ms}$. An asymmetrical pulse form was desired to address the possible non-linearity of the sensor response and ensure capability to observe the sensor performance both in the high and low $\mathrm{dB} / \mathrm{dt}$ magnetic fields.

a)
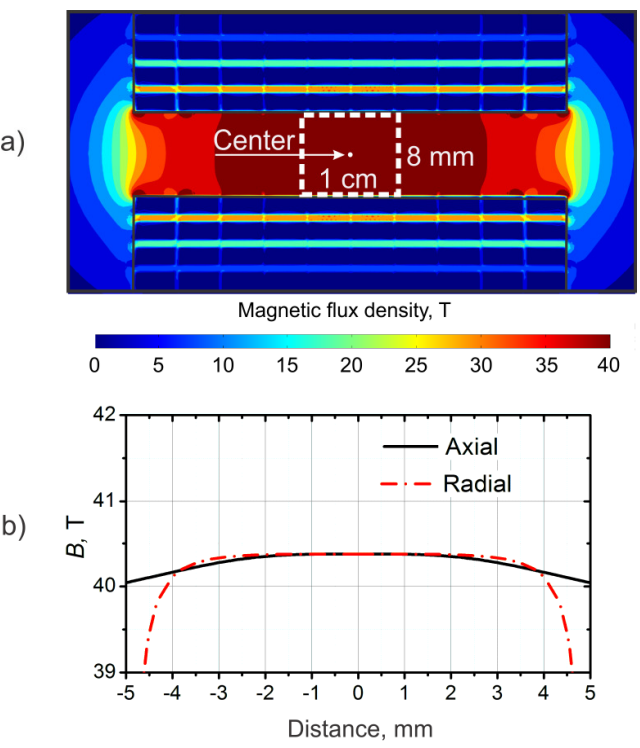

Fig.1. a) magnetic field distribution inside the $15.5 \mu \mathrm{H}$ inductor. White rectangle represents area with $99 \%$ magnetic field homogeneity b) axial and radial magnetic field profiles. 
One of the most convenient ways of generating magnetic field above $2 \mathrm{~T}$ is using the solenoid type inductors. We have chosen a multilayer enforced solenoid coil topology. These types of coils offer high experimental repeatability, considerable inner volume and durability [11]. The pulse form and the pulse duration can be adjusted by changing the number of windings and layers. Since the kA range current was commutated to generate high magnetic fields, the finite element method (FEM) analysis was used to estimate the magnetic field distribution dependence on the pulsed current. The magnetic field distribution inside the coil is shown in Fig.1.

Since the facility is designed for the calibration of the magnetic field sensors, the high homogeneity of the magnetic field distribution must be ensured. Based on FEM the structure of the coil and the required energy storage have been determined. The 40 windings 4 layer $(15.5 \mu \mathrm{H})$ enforced pulsed inductor (10 windings each layer) was introduced as a load. The inner and the outer diameters are $12 \mathrm{~mm}$ and $30 \mathrm{~mm}$, respectively. It was determined that 38 $40 \mathrm{kA}$ is required to generate the $40 \mathrm{~T}$ magnetic field in the proposed coil. Subsequently, the capacitor for energy accumulation has been evaluated. It has been determined that a capacitor in the range of $5-6 \mathrm{mF}$ is applicable. The application of a $5.5 \mathrm{mF}$ capacitor results in a pulse of $1.5 \mathrm{~ms}$ duration, which meets the requirements.

The solenoid has been wound using $\mathrm{Cu}-\mathrm{Nb}$ wire $(4.20 \times$ $2.37 \mathrm{~mm}^{2}$, strength UTS $=1.2 \mathrm{GPa}$ ), insulated with Kapton film and reinforced with Zylon fiber-epoxy composite. It consisted of 4 layers (10 turns in each layer, total of 40) with inner diameter of $14 \mathrm{~mm}$. The $12 \mathrm{~mm}$ steel shielding was implemented to prevent coil destruction due to the Lorentz forces in high magnetic fields. A second coil for magnetic fields up to $20 \mathrm{~T}$ with a nonmetallic outer casing made from polyamide material was also introduced to avoid tail of the magnetic field pulse [11]. The cooling time of the coils after the pulse is in the range of 3.5-4 $\mathrm{min}$ at room temperature for pulses up to $25 \mathrm{~T}$. For generation of the higher magnetic fields the active cooling using liquid nitrogen is required.

\section{B. Development of the pulse forming circuit}

The simplified circuit of the developed pulse forming circuit and the generator is shown in Fig.2.

As it has been mentioned above, in order to generate high magnetic field, the kA range current must be discharged through the inductor. In our case the high pulsed power thyristor SCR1 (voltage $4.2 \mathrm{kV}$, peak current $53 \mathrm{kA}$ ) has been chosen as the semiconductor switch to discharge the capacitor banks. The generator can generate pulses with the peak power up to $212 \mathrm{MW}$. Application of the thyristors is advantageous due to the possibility to switch high surge currents without the need of several parallel transistor switches. Nevertheless, the thyristors are more limited by the $\mathrm{dI} / \mathrm{dt}$. In our case the $\mathrm{dI} / \mathrm{dt}$ is limited by the inductance of the coil and the discharge capacitor, therefore a thyristor with typical $\mathrm{dI} / \mathrm{dt}=400 \mathrm{~A} / \mu \mathrm{s}$ is applicable.

As it can be seen from Fig.2. the high magnetic field generator consists of: 1) high voltage power supply; 2) capacitor bank; 3) measurement circuit; 4) high power switch with control circuit; 5) crowbar circuit; 6) reinforced inductor; 7) remote control. The $4 \mathrm{kV}$ power supply is shortcircuit proof using a custom made $600 \mathrm{~W}$ isolation transformer. The charging of the capacitor bank $\mathrm{C} 1$ is controlled using relay $\mathrm{K} 1.1$ and the voltage control circuit.

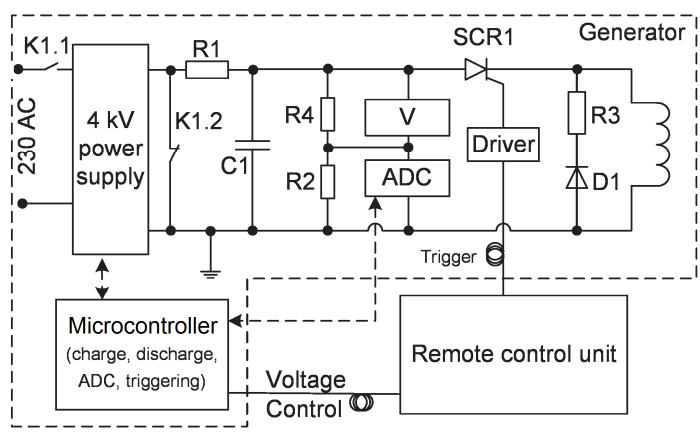

Fig. 2. Simplified circuit of the magnetic field generator.

The two low inductance $2.7 \mathrm{mF}, 4 \mathrm{kV}$ paper-oil capacitors have been used to form the $\mathrm{C} 1$ bank of $5.4 \mathrm{mF}$. The current limiting resistance $\mathrm{R} 1$ is used both for limitation of the charging current and as a load for the emergency discharge of the capacitors using high power relay K1.2. The comparator circuit has been implemented in the control unit, which drives the relay K1.1. The measurement results from the ADC (divider R2) are compared to the voltage set value. The charged capacitor array $\mathrm{C} 1$ is discharged through the load using the thyristor switch SCR1. The SCR is driven by an optically decoupled driver, which is triggered by the control circuit and the remote control, respectively.

The crowbar circuit has been implemented in the design (R3, D1) to introduce an asymmetrical pulse waveform, by increasing the fall time. Also the circuit is used for the compensation of the reverse-charge of the capacitor bank [12].

For safety reasons the remote control of the pulsed magnetic field generator has been developed. The operating principle of the control unit is shown in Fig.3.

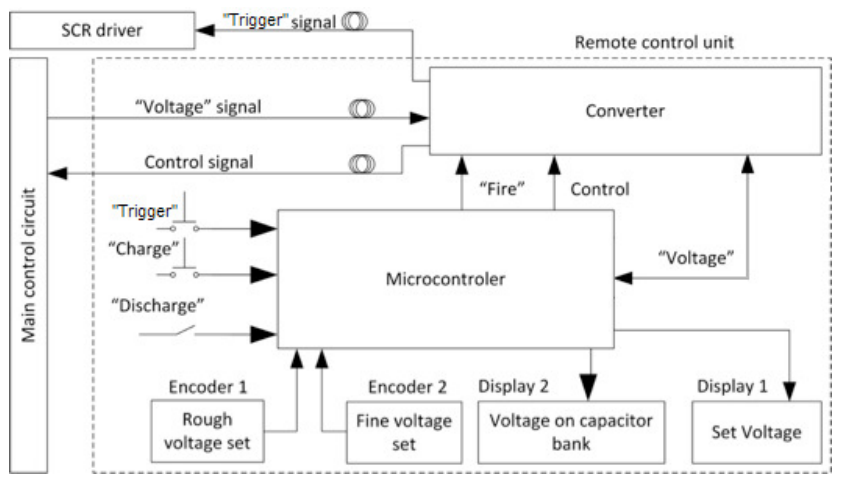

Fig.3. Block diagram of the remote control unit.

First, voltage set-point is set at the control unit and sent to the pulse generator. The capacitor charge, the discharge through the inductor coil or the emergency discharge through the ballast resistors are triggered with the corresponding switches. Voltage on the capacitor bank is constantly monitored and displayed for the user. 


\section{The generator}

The prototype of the magnetic field generator has been designed and fabricated. The photograph of the developed prototype and the remote control for safe operation are shown in Fig.4.

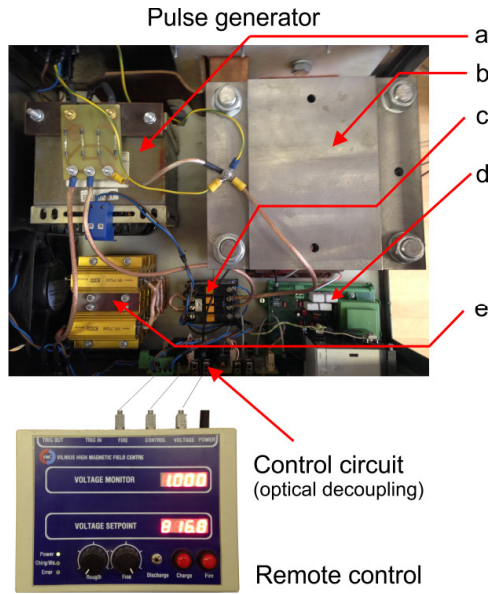

Fig.4. The prototype of the magnetic field generator unit and remote control unit: a) isolation transformer and rectification circuit; b) SCR/Crowbar diode circuit; c) relays for charge and discharge; d) driver circuit e) charge/discharge resistance.

The resulting generated magnetic field pulse waveform with a $15.5 \mu \mathrm{H}$ coil is shown in Fig.5.

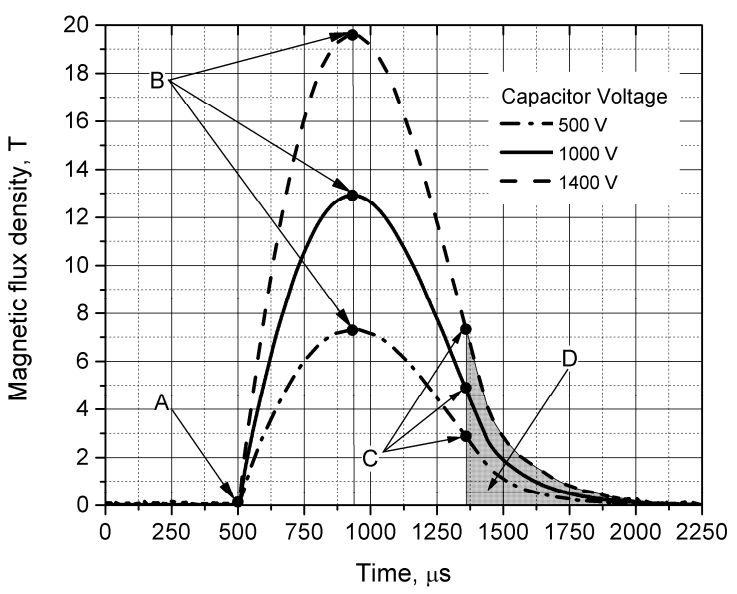

Fig.5. The pulse waveform of the developed pulsed magnetic field generator, where A - SCR turn-on, B - capacitor is discharged (current through inductor reaches the maximal value), $\mathrm{C}-\mathrm{SCR}$ is turned-off, D - the induced current is flowing in the crowbar/inductor circuit. The pulses have been generated at room temperature.

As it can be seen in Fig.5., the pulse features an asymmetrical shape. The magnetic field amplitude (Fig.5. B) can be controlled by the charging voltage, which ensures $\pm 1 \%$ pulse generation repeatability. The crowbar circuit, active resistance of inductor, and current that was stored in the case of coil after turning-off of SCR and D1 cause the asymmetrical shape of the pulse. The repetitive frequency of the pulses is limited by the Joule heating in the coil and the time that is required to charge the capacitor bank.

\section{RESUltS}

The facility has been applied for the calibration of the CMR-B-scalar magnetic field sensors. Previously, it was demonstrated that the CMR-B-scalar sensors based on the colossal magnetoresistance (CMR) effect in thin manganite films (La1-xSrxMnO3) [13] can be successfully used for the measurement of the pulsed magnetic field distribution in the bore of a coilgun [11] and in the vicinity of railgun rails [14]. However, the sensor's response is not a linear function in respect to the magnetic flux density $\mathrm{B}$. In addition, the sensor output signal is dependent on the ambient temperature. This requires calibrating the sensor over the operating temperature and magnetic field range.

The CMR-B-scalar sensor and a reference B-dot sensor, which was calibrated in advance, were placed in the center of the $15.5 \mu \mathrm{H}$ coil at approximately the same position. The magnetic field pulse of $25 \mathrm{~T}$ has been generated using the proposed setup. The signals of the CMR-B-scalar sensor (voltage drops across the sensor) and signals from the B-dot sensor were recorded by a digital oscilloscope. Then, the procedure was repeated in the temperature range $0-50{ }^{\circ} \mathrm{C}$ with a step of $3^{\circ} \mathrm{C}$. The data was processed using MATLAB routines to create the final calibration file. However, it should be noted that during the calibration of the sensors in pulsed magnetic field some problems can appear due to high $\mathrm{dB} / \mathrm{dt}$. The occurrence of the parasitic signal caused by the so-called "loop effect", the relaxation process in the sensor and the digitization step of the recording signals can decrease the accuracy of the calibration. To avoid these effects the magnetic field must change as slowly as possible. Therefore, in order to increase the accuracy of calibration, the falling part of magnetic field pulse (Fig.5.) was used $(\mathrm{dB} / \mathrm{dt}$ is smaller). The calibration procedure is summarized in Fig.6.
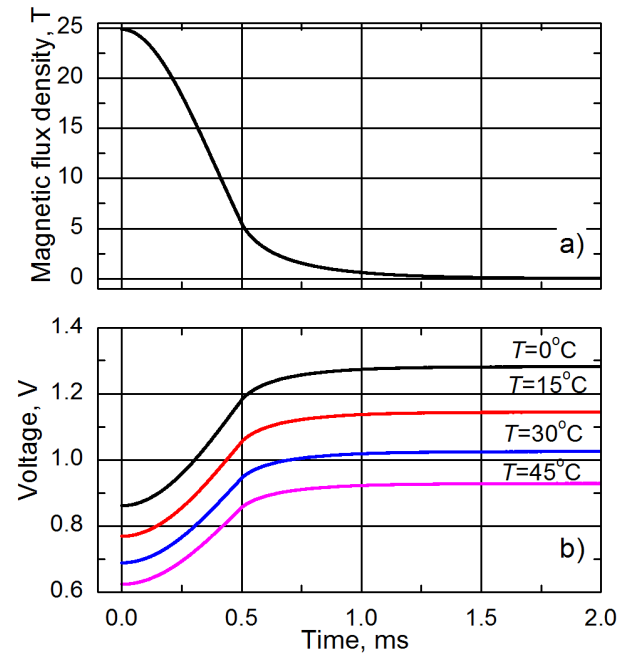

Fig.6. Calibration curves: a) magnetic field measured with B-dot sensor b) response signal of the CMR-B-Scalar magnetic field sensor.

The integrated signal from loop sensor Fig.6.a) and the output voltage across sensor Fig.6.b) are shown. It can be seen (Fig.6.b)) that the total signal across CMR sensor is actually a superposition of two components $U_{s}(t)=U_{0}-$ 
$\Delta U_{\mathrm{CMR}}(t)$. Here $U_{0}$ is the voltage across the sensor at zero magnetic field. This voltage depends on the temperature of the sensor and it is considered to be constant in the duration of the pulse. $\Delta U_{\mathrm{CMR}}(t)=\mathrm{f}(|B|)$ is a voltage decrease due to the magnetoresistance effect. Generally, the calibration table is created by assigning the $\mathrm{B}$ values from the reference sensor to the $\Delta U$ values from the CMR-sensor signal measured at the same point in time: $B\left(t_{\mathrm{i}}\right) \rightarrow \Delta U_{\mathrm{CMR}}\left(t_{\mathrm{i}}\right)$. The assignment $B(\Delta U)$ was made for each temperature (for each $U_{0}$ ) of calibration. Finally, a family of calibration curves $B(\Delta U)$ for different $U_{0}$ (which correspond to different temperatures) for a particular CMR-B-scalar sensor are stored to PC.

\section{CONCLUSIONS}

In this work a $43 \mathrm{~kJ}$ mobile pulsed magnetic field facility for single pulse calibration of magnetic field sensors has been developed. It is capable to generate magnetic field up to $40 \mathrm{~T}$. The proposed design is applicable for generating $0.3-2 \mathrm{~ms}$ pulses, with high repeatability. The proposed facility has been successfully tested experimentally for calibration of CMR-B-scalar magnetic field sensors.

\section{ACKNOWLEDGMENT}

This work was partly supported by the Agency for Science, Innovation and Technology (MITA) High-Tech Development Programme project MAGEPS (No. 31V-149).

\section{REFERENCES}

[1] Mapps, D.J. (1997). Magnetoresistive sensors. Sensors and Actuators A, 59, 9-19.

[2] Lenz, J., Edelstein, A.S. (2006). Magnetic sensors and their applications. IEEE Sensors Journal, 6 (3), 631649.

[3] Paun, M.A., Sallese, J.M., Kayal, M. (2012). Offset and drift analysis of the hall effect sensors. The geometrical parameters influence. Digest Journal of Nanomaterials and Biostructures, 7 (3), 883-891.

[4] Herlach, F. (2002). Laboratory electromagnets-from Oersted to megagauss. Physica B: Condensed Matter, 319, 321-329.

[5] Zherlitsyn, S., Wustmann, B., Herrmannsdorfer, T., Wosnitza, J. (2012). Status of the pulsed-magnetdevelopment program at the Dresden high magnetic field laboratory. IEEE Transactions on Applied Superconductivity, 22 (3), 4300603.
[6] Nagabhushana, S., Krishnan, S., Suryan, G. (1974) Generation of high magnetic fields using thyristors. IEEE Transactions on Industrial Electronics and Control Instrumentation, IECI-21 (4), 241-243.

[7] Krug, H. et al. (2001). The Dresden high-magnetic field laboratory - overview and first results. Physica B, 294-295, 605-611.

[8] Novickij, V., Grainys, A., Novickij, J., Lucinskis, A., Zapolskis, P. (2013). Compact microsecond pulsed magnetic field generator for application in bioelectronics. Elektronika ir Elektrotechnika, 19 (8), 25-28.

[9] Novickij, V., Grainys, A., Novickij, J., Lucinskis, A. (2014). Programmable pulsed magnetic field system for biological applications. IEEE Transactions on Magnetics, 50 (11), 5101004.

[10] Herrmannsdörfer, T., Krug, H., Pobell, F., Zherlitsyn, S., Eschrig, H., Freudenberger, J., Müller, K.H., Schultz, L. (2003). The high field project at Dresden/Rossendorf: A pulsed $100 \mathrm{~T} / 10 \mathrm{~ms}$ laboratory at an infrared free-electron-laser facility. Journal of Low Temperature Physics, 133 (1), 41-59.

[11] Liebfried, O., Loffler, M., Schneider, M., Balevicius, S., Stankevic, V., Zurauskiene, N., Abrutis, A., Plausinaitiene, V. (2009). B-scalar measurements by CMR-based sensors of highly inhomogeneous transient magnetic fields. IEEE Transactions on Magnetics, 45 (12), 5301-5306.

[12] Bernardes, J., Swindler, S. (2005). Modeling and analysis of thyristor and diode reverse recovery in railgun pulsed power circuits. In 2005 Pulsed Power Conference, 13-17 June 2005. IEEE, 79-82.

[13] Balevičius, S., Žurauskienè, N., Stankevič, V., Keršulis, S., Plaušinaitienè, V., Abrutis, A., Zherlitsyn, S., Herrmannsdörfer, T., Wosnitza, J., Wolff-Fabris, F. (2012). Nanostructured thin manganite films in megagauss magnetic field. Applied Physics Letters, $101(9), 92407$.

[14] Schneider, M., Liebfried, O., Stankevic, V., Balevicius, S., Zurauskiene, N. (2008). Magnetic diffusion in railguns: Measurements using CMR-based sensors. In 2008 14th Symposium on Electromagnetic Launch Technology, 10-13 June 2008. IEEE, 1-6.

Received June 30, 2015. Accepted September 30, 2015. 